\title{
A Review of Emerging Techniques on Generation Expansion Planning
}

\author{
Jinxiang Zhu \\ Student Member, IEEE \\ Mo-yuen Chow \\ Senior Member, IEEE \\ Department of Electrical and Computer Engineering \\ North Carolina State University \\ Raleigh, North Carolina 27695-7911
}

\begin{abstract}
Generation expansion planning is a challenging problem due to its large-scale, long-term, non-linear, and discrete nature of generation unit size. Since the computation revolution, there are several emerging techniques proposed to solve large scale optimization problem. Many of these techniques have been reported to use in generation expansion planning. This paper will describe these emerging optimization techniques, including expert systems, fuzzy logic, neural networks, analytic hierarchy process, network flow, decomposition method, simulated annealing, and genetic algorithm and their potential usage in solving the challenging generation expansion planning in future competitive environments in power industry. This paper provides useful information and resources for future Generation Expansion Planning.
\end{abstract}

Keyword: Generation Expansion Planning (GEP), Expert Systems (ES), Fuzzy Set Theory and Fuzzy Logic (FL), Artificial Neural Networks (ANN), Analytic Hierarchy Process (AHP), Network Flow (NF), Decomposition Methods, Simulated Annealing (SA), Genetic Algorithm (GA)

\section{Introduction}

Generation expansion planning (GEP) is to determine WHAT generating units should be constructed and WHEN generating units should come on line over a long-term planning horizon. The criteria are to minimize the total cost and/or maximize the reliability with different type of constraints. The total cost is the sum of capital investment and operation cost. The constraints include capacity constraints, energy constraints, and operation constraints etc. Generally GEP is a non-linear integer programming problem which can be solved by linear programming, nonlinear programming, dynamic programming, and integer programming techniques with certain simplifications. These mathematical models have been reviewed in [1]. This paper will focus on several potential emerging techniques that have becn applicd in GEP.

Since the emerge of power systems, engineers have accumulated significant amount of experience to GEP. The

PE-649-PWRS-0-01-1997 A paper recommended and approved by the IEEE Power System Engineering Committee of the IEEE Power Engineering Society for publication in the IEEE Transactions on Power System. Manuscript submitted September 23, 1996; made available for printing January 16, 1997. experience can be translated to a large set of rules. Expert systems (ES) have been developed based on these valuable rules to solve the GEP problems $[2,3,4]$. ES have advantages on performance, consistence, reproducibility, and lower costs. The ES knowledge usually is based on quantitative data. However there is knowledge which cannot be described by quantitative but qualitative data. ES are not suitable to handle qualitative data nor it is a robust tool in uncertainty environments [5]. Fuzzy set theory and fuzzy logic are more appropriate tools to incorporate qualitative data into decision making process $[6,7,8,9]$.

Artificial neural network is a potential tool for optimization [10]. Hopfield Networks and Boltzmann Machines have been employed for classification and solving combinatorial optimization problems [11]. GEP can be firstly formulated as an integer programming problem or a mixed integer programming problem subject to a number of equality and inequality constraints, then a Hopfield network can be applied to solve the GEP problem, which will be introduced in the later section.

Since non-linearity and discrete nature in GEP, the problem becomes a large-scale nonlinear integer programming problem. Non-linear programming or integer programming is difficult to solve. For a large-scale nonlinear integer programming GEP problem, it is much more difficult to solve by mathematical techniques directly. One of the promising methods to solve GEP is to break down the problem into smaller, simpler subproblems by decomposition principles. GEP model can be decomposed by its model structures or its mathematical functions $[12,13,14]$. Bender's decomposition and Dantzig-Wolfe decomposition are two popular decomposition methods. Bender's decomposition can decompose GEP into a planning master problem and an operation subproblem. The planning master problem is an integer programming problem while the operation subproblem is a non-linear programming problem $[12,14]$. The coordination of the master problem and subproblem is to minimize the total cost.

Analytic hierarchy process considers the long-range planning process from the point of view both of projecting forward from the current position to define a possible future state, and of identifying desirable future states and working backwards from those to be reached [23]. The process continues till forward and backward looking processes are consistent with each other. The hierarchy method provides 
insight of the problem and to explicitly assess the impact of multiple objectives in the long-range planning. It leads to a continuous revision and improvement of planning and the beliefs about outcomes. Clarke et al. have applied AHP to GEP in [24].

Network Flow Theory has been applied to optimization problems for power system operation and planning, such as, hydrothermal scheduling $[25,26,27,28]$ and resource planning [29]. These problems can be best analyzed by means of graphical or network representations. Most cases are modeled as the minimum cost network flow problems which can be solved by linear programming, non-linear programming, and special network flow algorithms.

As mentioned previously, GEP is a large-scale optimization problem with non-linearity and discrete nature. The analytic algorithms are difficult to find the optimal solutions. Genetic algorithm and simulated annealing are two promising heuristic optimization techniques to solve large-scale non-linear integer programming problems. These techniques can avoid trapping in local minima and in many cases obtain better solutions than other heuristic approaches [16,17,18,19,20,21,22].

The following sections will describe several emerging optimization techniques such as expert systems, fuzzy logic, neural networks, analytic hierarchy process, network flow, decomposition method, simulated annealing, and genetic algorithm with their potential usage in solving this challenging GEP problem in the future competitive environment of power industry.

\section{Techniques on Generation Expansion Planning}

\subsection{Expert Systems:}

Expert systems are programs reconstructing the expertise and reasoning capabilities of qualified specialists within limited domains. The preliminary basic assumption is that experts construct their solutions from single piece of knowledge which they select and apply in a suitable sequence. Hence expert systems require detailed information about the domain and the strategies for applying these information to problem solving. In order to construct an expert system, the knowledge must be formalized, represented, manipulated according to some problem solving method. The knowledge is represented by rules like "If $X$ then $Y$ " or objects and states. The problem solving methods are highly parametrized algorithms which are rule or object-based programming. Therefore expert systems derive their knowledge from expert and provide the decision based on the inputs. Generation expansion planning is a highly knowledge intensive area which can be solved by combining heuristic knowledge with numerical analysis and simulation. The expertise is developed based on historical planning experience, the effective use of analytical methods, a clear understanding of the interrelation among the parameters, and how to integrate their results into coherent designs. The expert systems can help to consider the detailed models and to find the solutions efficiently. $[2,3]$ gave a review of expert systems in power system planning and operation. Expert systems have advantages on performance, reproducibility, consistence, and low cost. But human experts are much more creative and innovative than any expert system. Expert systems have been applied to GEP areas $[2,3,4]$.

David and Zhao integrated an expert system into a dynamic programming based generation expansion planning program [4]. The expert system was used to incorporate a number of rule-based procedures in the decision making part of the dynamic programming algorithm. This allowed some practical engineering considerations to be imposed on decision making at each stage. The expert system can prune out of unsuitable states and impermissible transition of dynamic programming based on the rules from engineering experiences.

\subsection{Fuzzy Set Theory / Fuzzy Logic}

Fuzzy set theory was first proposed by Zadeh in 1965. Based on the results of numerical analysis, the relationships of the inputs and outputs are identified as fuzzy rules. That is, the inputs are fuzzified based on their membership functions; the relationship of inputs and outputs are described by fuzzy rules; the outputs are defuzzified to be a crisp output based on the output membership function. This is typical process used in fuzzy logic. The outputs can be fine tuned by modifying fuzzy rules and membership functions. $[6,7,8]$

Dhar was the first to apply fuzzy set theory in power systems [9]. He introduced the (fuzzy / non-fuzzy) utility to describe the alternatives' impacts on fuzzy system states. Based on the utility matrix for a criterion of merit and the given fuzzy system states, he determined the fuzzy utility sets associated with each alternative (the minimum of the membership grade of utility value from fuzzy system states for one alternative and utility value membership grade). Fuzzy sum operation is used for absorbing redundant states. The maximal grade of fuzzy utility set for an alternative is assigned to this alternative. The alternative which has the highest grade is the optimal solution for the given criterion.

Handke et al. [6] used the pseudo fuel price to coordinate the long- and short-term generation planning. In their approach, six criteria are introduced for each unit. The final rating of each unit was defined by the weighted average of the accomplishment of criteria for it. Each criterion value and its weighting factor were then fuzzified based on the corresponding membership functions. Extension principle was applied to the fuzzy ratings of the criteria and the weighting factors to get the membership value for the final rating. Then the preference membership value for this unit against the average of other units is 
obtained by fuzzy ranking algorithms. Then the preference is defuzzified to provide a crisp preference. Finally the pseudo fuel price is defined as the normalized difference of the preference of this unit and the maximum preference.

Tanabe et al. presented an efficient algorithm for linguistic risk assessment of uncertain factors in generation planning [7]. The correlation coefficients of the multiple objectives and the uncertainties on objectives' impacts are evaluated and fuzzified to linguistic values based on the system analysis. The linguistic possibility for deviation of each uncertain factor and linguistic weights of objectives are assessed by a Decision Maker. The aggregated risks of uncertain factors are evaluated for all objectives. On the other hand, the linguistic assessments and linguistic truth are integrated by the truth value to construct the membership function of linguistic expressions. The linguistic expression which is closest to the aggregated risk in Hamming distance is then selected.

David and Zhao [5] incorporated fuzzy set theory in the expert system for optimal generation planning. Judgment or preference is difficult to represent as a deterministic or probabilistic constraint. This qualitative judgment is natural to be described by linguistic rules; therefore, fuzzy set theory was involved in the decision making system of expert systems. The use of fuzzy set theory significantly reduce the decision space.

\subsection{Artificial Neural Networks}

The advent of artificial neural networks (ANNs) provides another tools to solve generation expansion planning problem. ANNs possess the ability to perform pattern recognition, prediction, and optimization in a fast and efficient manner after appropriate training. A neural network is a network with nodes or neurons analogous to the biological neurons. The nodes are interconnected through weighted links. The weights are usually adjustable and can be trained through a learning algorithm and training examples. Multi-layer feed-forward networks are trained off-line using supervised learning and are used to perform pattern recognition, prediction and classification in an online environment. Kohonen networks are trained by unsupervised learning and used for classification. Hopfield Networks and Boltzmann Machines are trained by unsupervised learning algorithm and are employed for classification and solving combinatorial optimization problems [10]. The major drawback of the Hopfield network is the limited storage capacity of the associative memory. Since there exist local minima in the energy function, the final solution is good but not necessarily optimal. One possibility to suppress the convergence to undesired local minima is by adding probabilistic noise and incorporating simulated annealing techniques to the continuous Hopfield networks. The Boltzmann Machine adds hidden units to Hopfield architecture and update the output vector only with the probability of Boltzmann Gibbs distribution.

Generation Expansion Planning has been formulated as a $0-1$ integer programming problem and then mapped onto the modified Hopfield neural network [11]. Suppose GEP is expressed by $0-1$ integer programming problem:

$$
\text { Minimize: } f(\mathrm{v}) \quad \mathrm{V}=\left(V_{1}, \ldots, V_{N}\right),
$$

$$
\text { Subject to: } \quad h_{j}(\mathrm{~V}) \geq 0 \quad j=1, \ldots, M,
$$

where $\mathbf{V}$ denotes the $\left(\begin{array}{lll}N & x & l\end{array}\right) 0-1$ variable vector and $f(v)$ is a linear function. This GEP model is solved by the modified Hopfield neural network as show in Fig. 1.

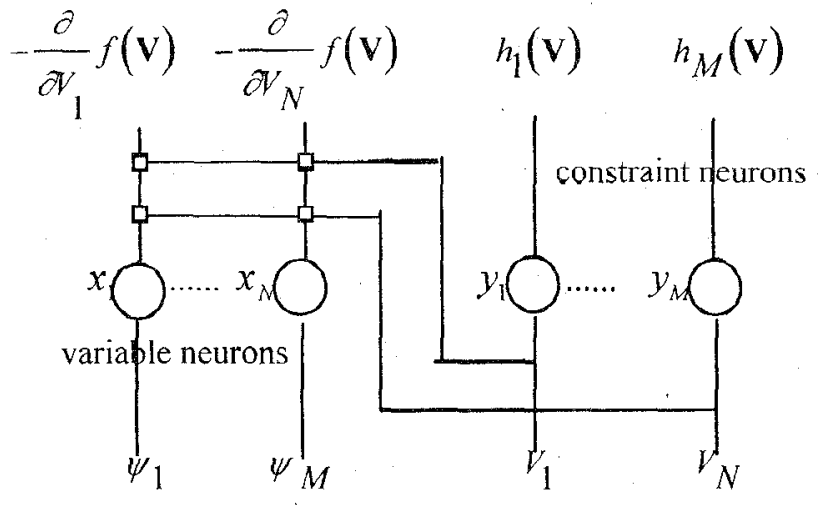

Fig. 1 Hopfield neural network structure for GEP

口 weighting factor $T_{i j} i=I, \ldots, N, j=I, \ldots, M$,

$x_{i}$ the input-output function (sigmoid functions) of variable neuron $i$,

$y_{j}$ the input-output function of constraint neuron $j$,

$T_{i j} \quad$ the connection from constraint neuron $j$ to variable neuron $i$,

$\psi_{j} \quad$ the output of constraint neuron $j$.

The variable and constraint neurons have the following relationships:

$$
\left.\begin{array}{lr}
\frac{d u_{i}}{d t}=-\frac{\partial}{\partial V_{i}} f(\mathrm{v})+\sum_{j=1}^{M} T_{i j} \psi_{j}, & i=1, \ldots N, \\
V_{i}=x_{i}\left(u_{i}\right), & i=1, \ldots N,
\end{array}\right\}
$$

where $u_{i}$ the input of the variable neuron $i$.

$$
\text { If } \frac{d u_{i}}{d t}=\frac{\partial E}{\partial V_{i}}, \text { then }
$$




$$
E=f(\mathbf{v})+\sum_{i=1}^{N} \int \sum_{j=1}^{M} T_{i j} \cdot y_{j}\left(h_{j}(\mathrm{v})\right) d V_{i}
$$

It can be proved that if the input-output neurons are monotonically increasing, the energy function $E$ converges to one of its minima with time [61]. However the network cannot guarantee the optimal solution.

For a GEP problem, the objective is to minimize the sum of fixed cost and variable cost, subject to the capacity constraints, spinning reserve constraints, and the limits of new installation units [11]. The weighting factors are predetermined based on the relationship between constraints and decision variables. This modified Hopfield neural network can be used to solve GEP problem by an iterative scheme. Initial states of units are assumed to be given at the beginning of the scheme: (i) randomly update the decision variable neurons' inputs by

$$
u_{i}^{t+1}=u_{i}^{t}-\frac{\partial}{\partial V_{i}} f(\mathbf{v})+\sum_{j=1}^{M} T_{i j} \psi_{j},
$$

(ii) calculate the output of the corresponding decision variable neurons and then update the all constraints neurons; the scheme repeat until all the constraints are satisfied and decision variables converge.

\subsection{Network Flow Theory}

Network Flow Theory has been applied in power systems in many areas such as hydrothermal scheduling $[25,26,27,28]$, and resource planning [29]. Since electric transmission, distribution networks, and hydro systems are physically in network flow nature, the minimum cost network flow model fits easily in these optimization problems. Sometimes operation optimization problem is decomposed into hydro subproblem and thermal subproblem.

Hydrothermal scheduling problem aims to determine hydro and thermal generation at each moment minimizing the total system operational cost $[25,26,27,28]$. This is complex non-linear, non-differentiable, stochastic, largescale, dynamic problem which can be formulated as a nonlinear capacitated network flow problem. [25] applied a second-order network flow algorithm into hydrothermal scheduling problem based on the Truncated Newton method. The proposed algorithm takes advantage of the three-diagonal structure of the Hessian matrix, to significantly reduce computation effort. [26] considered spinning reserve in hydrothermal scheduling through network flows. A coupled network formulation of hydrothermal scheduling was proposed to impose system load and spinning reserve constraints on both hydro and thermal generation and directly minimize thermal production costs without decoupling the problem into hydro and thermal subproblem. Franco et al. decomposed the hydrothermal scheduling into hydro and electric subproblems and considered hydraulic coupling and transmission limits in [27]. $\mathrm{Li}$ et al. implemented incremental network flow programming into hydrothermal coordination [28].

Nabona employed the multi-commodity network flow technique to the long-term hydro-generation optimization [29]. For the long-term hydro-generation optimization, the water inflows are not deterministic but stochastic. This paper proposed a network flow model to minimize the total expected production cost, by taking into account the stochastic water flows. The multi-commodity means that water inflows are represented as multi-amount of water with different probability of occurrence. The expression of the expected production cost in terms of flows on the replicated network of each commodity is developed. Therefore, the long-term hydro-generation problems can be reduced to multi-commodity network flow problems on one or several unconnected networks, with or without side constraints.

\subsection{Analytic Hierarchy Process}

Analytic hierarchy process (AHP) is a two-point boundary problem, from present to future and from future back to present, with the following management: give the present set of policies and a desired future, modify the existing policies and design new compatible policies to attain the future. AHP then modifies the desired future for greater compatibility with the effectiveness of new policies. Iteration terminates when a good feasible solution arises, i.e., the forward and backward looking processes are consistent with each other.

In [24], the overall goal of GEP is to choose the best resource plan, and it is placed at the top of hierarchy. The factors affect the choice of the best resource plan are divided into three genetic groups: (i) the external business environment, (ii) the internal business environment, and (iii) decision maker's objectives. Each group is evaluated by several attributes / criteria associated with the relative importance under each risk scenario. The most important factors are then selected from both the external and internal business environments in order to construct a reduced value tree. The preference of each alternative resource plan is elicited in respect to each scenario, based on the reduced value tree. Finally, the overall ratings of the plans are calculated based on the probability and the rating in each scenario.

AHP provides insight of the problem and assess explicitly the impacts of different issues in planning. It leads to a continuous revision and improvement of planning and the beliefs about outcomes. AHP has advantages to deal with multi-objective decision making problems. This method requires that the objectives are mutually preferentially independent. Adding another alternatives may change the ranking of the original alternatives.

\subsection{Decomposition Method:}

It is difficult to solve a large scale problem directly because of excessive computer storage memory and 
execution time. In order to make the execution of the large scale problem more efficient, most approaches divide the problem into smaller subproblems and adjust the optimal solutions of the subproblems to produce an optimal solution for the large scale problem. Dantzig-Wolfe decomposition and Benders decomposition are two popular methods in GEP area. Decomposition method actually is an iterative process to find the optimal marginal conditions. Dantzig and Wolfe developed a decomposition principle imposing on each subproblem additional constraints so that the optimal solution of one subproblem is independent from the solutions of the other subproblems. If the feasible region is a closed convex polyhedron and the objective function is linear, Dantzig and Wolfe decomposition is applicable. Additional constraints are selected so that the union of the solutions of the subproblems will be optimal for the largescale problem. How to split the right hand-side constants of the additional constraints to each separated part is the key issue in Dantzig-Wolfe decomposition. It can be shown that finite convergence of the algorithm when the number of possible contingencies is finite. Benders decomposition assumes that the results of master problem are known. Therefore, the remaining unknown part is a smaller subproblem which can be solved easily. The original objective function of the large scale problem is considered in the master problem except that the objective function part corresponding to the subproblem is replaced by the optimal objective function of the subproblems. When the master problem is solved and different results are obtained, the subproblem has to be reentered to update its optimal solution. The procedure is terminated when the solutions of master problem can not be improved any more. Since the continuous operation variables and integer investment variables are linearly separable in generation expansion planning problems, it can be shown that the Benders decomposition methods are convergent [12].

Avriel, Dantzig, and Glynn decomposed the model into two parts: the first part represents the deterministic expansion planning, the second part represents the scenario simulations associated with probability of occurrence [13]. They used the importance sampling method to filter out most of the scenarios in Monte Carlo simulations and to estimate the average information about the scenarios to improve the deterministic part of the expansion planning. Parallel processing techniques are used to improve the computation efficiency for decomposition and importance sampling. Benders decomposition algorithm, stochastic linear programming, and dynamic programming are involved in this proposed method. Entriken further considered reliability analysis in GEP [14]. He decomposed problem into a reliability master program and a resource planning sub-program by Dantzig-Wolfe decomposition. Bender's decomposition is then applied to the resource planning subproblem as [13] does.

Noonan and Giglio [9] proposed a Non-Linear Mixed Integer Programming model for long-range generation expansion planning. The investment and operation cost, thermal/hydro generation, discounted factor, reliability constraints, discrete generation unit size, capacity constraints, and pumped hydro renewal constraints are considered in this model. Benders decomposition method is employed to decompose the model into mixed integer linear programming model for the investment master problem and non-linear programming model for operation subproblems. The analytic reliability model is approximated by a polynomial on the maximum allowable risk for the system. The master problem is a mixed integer linear programming problem by replacing the reliability constraints with all sets of linearized constraints that have been generated from the last stage operation subproblem. The operation subproblem is solved under the investment plan from the master problem. A commercial software package is used to solve the mixed integer linear programming. Baughman et al. [12] proposed a comprehensive electrical system planning method to incorporate transmission planning into integrated resource planning. The model is a mixed integer, nonlinear, stochastic programming problem and is solved by using generalized Benders decomposition (GBD).

\subsection{Simulated Annealing}

Simulated Annealing (SA) is essentially a simulation of annealing process of molten metals [15]. The iteration number used in SA is analogous to temperature level. In each iteration, a candidate solution is generated. If this solution is a better one, then it is accepted and used to generate another candidate solution. If it is a deteriorated solution, it is accepted when its probability of acceptance (Boltzmann distribution) is greater than a random number between 0 and 1 . In the next iteration, temperature is reduced according to some function of system states. The deteriorated solution is accepted with less and less probability through the iterations. The solution process continues until the maximum number of iteration is reached or the optimal solution is found. SA can find slightly better solution than some other heuristic method (e.g. sequential algorithm) $[15,16]$.

Wong et al. applied SA to deal with multiple fuels, multiple pollutants and highly non-linear heat rate functions in generation dispatch [16]. He also proposed a Parallel SA to efficiently solve short-term hydrothermal scheduling with reservoir volume constraints [17]. Chen and Liu used SA to solve a multi-objective and nondifferentiable VAR planning in power systems [15]. The advantages of SA approach lie in that it is capable of not only handling a mixed-integer nonlinear programming but also searching toward a global optimal solution. [18] considered multi-objective in VAR planning. SA is used to find the global non-inferior solution in first stage. Based on the first stage results, the primary objective function is chosen and the other objectives are transformed to constraints using trade-off tolerances in the second stage. The global optimal solution of this singleobjective problem is found by SA. 


\subsection{Genetic Algorithm:}

Genetic Algorithm (GA) is a random search procedure based on the survival of fittest theory [19]. A population of candidate solutions is first initialized subject to certain constraints. Each trial solution is then coded as a vector, termed a chromosome. A finite range of values for variables is selected and the minimum possible value in the range represented by string 0 's, with the maximum value represented by string l's. The desired degree of precision indicates the appropriate length of the binary coding. Each chromosome in the population is decoded into a form that is appropriate for evaluation. A fitness score is then assigned according to the objective. Each chromosome is assigned a probability of reproduction so that its likelihood of being selected is proportional to its fitness relative to other chromosomes in the population. According to the probability of reproduction, a new population of chromosomes is generated by probabilistically selecting strings from the current population. The selected chromosomes generate offspring via the use of specific genetic operators, crossover and bit mutation. The probability of crossover and bit mutation range from 0.6 to 0.95 and 0.001 to 0.01 , respectively. The process is halted if a suitable solution has been found, or if the available computing time has exceeded. Otherwise the new chromosome are scored and the cycle is repeated. Although there is no mathematical proof or guarantee that solution is globally optimal, it is most likely so [20,21]. However, the approach requires tremendous computing time.

Fukuyama and Chiang used genetic algorithm (GA) for the optimal long-range generation expansion planning [22] because of its ability and efficiency to find the global optimal solutions and its high suitability for parallel computation. The problem was formulated as a combinatorial optimization problem that determines the order of introducing generation units at each interval of the year. The total population is split into sub-population, which is allocated to a process on which conventional GA is applied. At every epoch, some strings are immigrated from the neighboring subpopulation, which can be installed on transputer. For a typical expansion problem (four technologies and five intervals), the Parallel GA method was shown more effective comparing with the dynamic programming method [22]. The more processors the system uses, the less the computation time is required, but the more cost is needed. The number of processors used is a trade-off between computation speed and cost.

Iba applied GA in Optimal Power Flow problem [14]. GA carried multiple path searching to avoid local minima. Artificial intelligent type "If-then" rules were applied statistically to guide the search. Multiple objectives are considered simultaneously by weighting factor method. It took more computing time than traditional methods for large systems.

\section{Conclusions}

The emerging technologies, such as simulated annealing, genetic algorithm, fuzzy logic, neural networks, expert systems, network flow theory, and analytic hierarchy process are powerful to solve large scale generation expansion planning problems. This paper describes the basic procedure for each emerging technique and its application on GEP. The merits and demerits are summarized. Due to the page limitation, the authors would apologize to those whose papers are not included.

\section{References}

[1] Jinxiang Zhu and Mo-yuen Chow, "A Review of Mathematical Modeling on Generation Expansion Planning", submitted to IEEE Trans. on PS.

[2] N. J. Balu, R. Adapa, G. Cauley, M. Lauby, and D. J. Maratukulam, "Review of Expert Systems in Bulk Power System Planning and Operation", Proceedings of the IEEE, Vol. 80, No. 5, May 1992.

[3] F. D. Galiana, D. T. McGillis and M. A. Marin, "Expert Systems in Transmission Planning", Proceedings of the IEEE, Vol. 80, No. 5, May 1992.

[4] A. K. David and R.D. Zhao, "Integrated expert systems with dynamic programming in generation expansion planning", IEEE Trans. on PS, Vol. 4, No. 3, 1989.

[5] A.K. David and R. Zhao, "An Expert System With Fuzzy Sets For Optimal Planning", IEEE Trans. on PS, Vol. 6, No. 1, February 1991.

[6] J. Handke, E. Handschin, K. Linke, H.-H. Sanders, "Coordination of Long- and Short-term Generation Planning in Thermal Power Systems", IEEE Trans. on PS, Vol. 10, No. 2, May 1995

[7] Ryuya Tanabe, Keiichiro Yasuda, Ryuichi Yokoyama, "An Algorithm for Linguistic Risk Assessment of Uncertain Factors in Generation Planning", Electrical Engineering in Japan, Vol. 115, No. 3, 1995.

[8] H. Satoh and Y. Serizawa, "Fuzzy Decision-Making on Electric Energy Strategy For Long-Term Generation Expansion Planning", IFAC Power Systems and Power Plant Control, Seoul, Korea, 1989.

[9] S. B. Dhar, "Power System Long-Range Decision Analysis Under Fuzzy Environment", IEEE Trans. on PAS, Vol. PAS-98, No. 2, March/April 1979.

[10] Kit Po Wong, "Artificial Intelligence and Neural Network Applications in Power Systems", IEE Second International Conference on advances in Power System Control, Operation and Management, Hong Kong, December 1993.

[11] Sasaki H., Kubokawa J., Watanabe M., Yokoyama R., and Tanabe R., "A solution of generation expansion problem by means of neural network", Proceedings First International Forum on Applications of Neural Networks to Power Systems, Seattle, 1991. 
[12] M. L. Baughman, S.N. Siddiqi, and J. W. Zarnikau, "Integrating Transmission into IRP, Part I: Analytical Approach; Part II: Case Study Results" , IEEE Trans. on PS, Vol. 10, No. 3, August 1995.

[13] M. Avriel, G. B. Dantzig, and P. W. Glynn, "Decomposition and Parallel Processing for Large-Scale Electric Power System Planning Under Uncertainty",

[14] Robert Entriken, "Parallel Decomposition of TwoStage Stochastic Linear Programs", Proceedings of the Workshop on Resource Planning Under Uncertainty for Electric Power Systems, Stanford University, January 21 22, 1989.

[15] Y. L. Chen and C. C. Liu, "Interactive Fuzzy Satisfying Method for Optimal Multi-Objective VAR planning in Power Systems", IEE Proceedings Generation, Transmission, Distribution, Vol. 141, No. 6, November 1994.

[16] Kit Po Wong, Bei Fan, C.S. Chang, A.C. Liew , "Multi-Objective Generation Dispatch Using Bi-Criterion Global Optimization", IEEE Trans. on PS Vol. 10, No. 4, November 1995.

[17] Kit Po Wong and Yin Wa Wong, "Short-Term Hydrothermal Scheduling with Reservoir Volume Constraints, Part I, II", IEE Second International Conference on Advances in Power System Control, Operation and Management, Hong Kong, December, 1993.

[18] Ying-Tung Hsiao, Hsiao-Dong Chiang, Chun-Chang Liu, and Yuan-Lin Chen, "A Computer Package for Optimal Multi-Objective VAR Planning in Large Scale Power System", IEEE Trans, on PS, Vol. 9, No. 2, May 1994.

[19] David B. Fogel, "An Introduction to Simulated Evolutionary Optimization" , IEEE Trans. on Neural Networks, Vol. 5, No. 1, January 1994.

[20] Kenji Iba, "Reactive Power Optimization by Genetic Algorithm", IEEE Trans. on PS, Vol. 9, No. 2, May 1994.

[21] Kit Po Wong and Yin Wa Wong, "Combined Genetic Algorithm / Simulated Annealing / Fuzzy Set Approach to Short-Term Generation Scheduling with Takeor-Pay Fuel Contract", IEEE Trans. on PS, Vol. 11, No. 1, February 1996.

[22] Yoshikazu Fukuyama and Hsaio-Dong Chiang, "A Parallel Genetic Algorithm for Generation Expansion Planning", International Conference on Intelligent System Application on Power System: ISAP '94, Montpellier, France, September 1994.

[23] J. R. Emshoff and T. L. Saaty, "Application of analytic hierarchy process to long range planning processes", European Journal of Operational Research, 10, 131-143, 1982.

[24] R. R. Clarke and P. Kleindorfer, "Choosing an Integrating Resource Plan for Electric Utilities: An Analytic Hierarchy Approach".

[25] G. G. Oliveira and S. Soares, "A Second-Order Network Flow Algorithm for Hydrothermal Scheduling", IEEE Trans. on PS, Vol. 10, No. 3, August 1995.
[26] F. J. Heredia, N. Nabona, "Optimum Short-Term Hydrothermal Scheduling with Spinning Reserve through Network Flows", IEEE Trans. on PS, Vol. 10, No. 3, August 1995.

[27] P.E.C. Franco, M.F. Carvalho, and S. Soares, "A Network Flow Model for Short-Term Hydro-Dominated Hydrothermal scheduling Problems", IEEE Trans. on PS, Vol. 9, No. 2, May 1994.

[28] C.A. Li, P.J. Jap, and D. L. Streiffert, "Implementation of Network Flow Programming to the Hydrothermal Coordination in an Energy Management System", IEEE Trans. on PS, Vol. 8, No. 3, August 1993.

[29] N. Nabona, "Multicommodity Network Flow Model for Long-Term Hydro-Generation Optimization", IEEE Trans. on PS. Vol. 8, No. 2, May 1993.

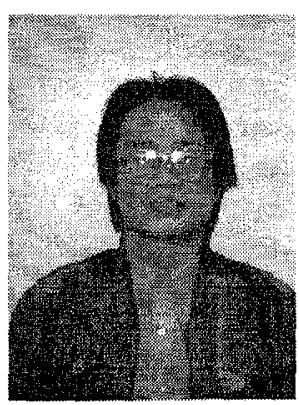

Jinxiang Zhu received his B.S. degree from Tsinghua University in Beijing PRC, and his M.S. degree from North Carolina State University in Electrical Engineering, respectively in 1990 and 1994 . Currently he is $\mathrm{Ph}$. D candidate student in North Carolina State University. He is student member of IEEE. His research interests include power system operation and planning, optimization techniques, fuzzy logic, and neural networks.

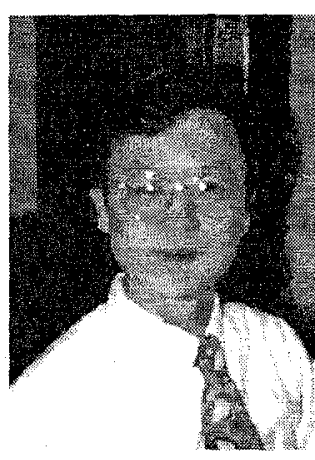

Mo-yuen Chow earned his B.S. degree at the University of Wisconsin-Madison (1982); M. Eng. degree (1983) and Ph.D. degrees at Cornell University (1987), all in Electrical Engineering. Upon completion of his Ph.D., Dr. Chow joined the faculty of North Carolina State University in Raleigh, NC, where he is presently an Associate Professor in the Department of Electrical and Computer Engineering. Dr. Chow has also worked as a consultant for OTIS Elevator Co., Taiwan Power Company, J.W. Harley Company, and as a faculty intern at Duke Power Company.

Since 1987, Dr. Chow has been working as a Principal Investigator in several projects in the areas of system monitoring, fault detection and control, applications of artificial neural network and fuzzy logic to power engineering. Dr. Chow served as a guest editor of IEEE Transactions on Industrial Electronics Special Issue on Application of Intelligent Systems to Industrial Electronics. $\mathrm{He}$ is . currently an Associate Editor of the IEEE Transactions on Industrial Electronics. He is also listed in Who's Who in Asian Americans. 